\title{
Depression in Elderly Life: Psychological and Psychosocial Approaches
}

\section{Shilpi Tripathi*}

Saw Swee Hock School of Public Health, National University of Singapore \& National University Health System, Singapore

*Corresponding author: Shilpi Tripathi, Saw Swee Hock School of Public Health, National University of Singapore \& National University Health System, 12 Science Drive 2, \#10-01, 117549, Singapore, Tel: +65-81213710

\begin{abstract}
Life depression at a later age due to its devastating effects is an important area of public health research. The elderly life depression linked with morbidity, deteriorating physical health, likelihood of suicide attempts and social cognitive functioning. All of which, in turn, are linked to increased life loss $[1,2]$. Depression tends to be less common in older adults than in earlier lifespan. All aspects of the older adult's lifetime condition are risk, protective factors, health, etiology, and outcomes. The impact of depression on older people is entirely different from younger people. However Effective treatment of older adults with depression, it often occurs with other medical illnesses and disabilities and it doubles an elderly person's risk of cardiac problems and increases their risk of death from this illness [3]. Many research studies shown that the presence of depression increases the chances of death and depression reduces an elderly person's ability to recover. This paper will explore an overview of the psychological theories, factors related to risk, prevention and late life treatment of depression. The possible cause of late depression is an individual's response with experiences during losses of loved ones and stressful life events [4].
\end{abstract}

\section{Depression Causes and Theories}

\section{The psychodynamic theory}

Freud wrote on the theory of the loss of loved ones the psychodynamic view of depression. Deprived of this view of depression, therapists believe that the root of depression is the loss of a person or an object. Freud believed that depressed people develop feelings of low self-esteem [5] and they are beginning to believe that they are responsible for the loss. Freud considered the failure to be the fault of him or her. Freud also claimed that feelings of extreme self-dislike emerge from the thoughts of the depressive, and as a result the depressive feels no real value to it-self and loses its self-esteem. Not feeling good about themselves starts an individual's down the path of depression [6]. According to many psychodynamic theorists that Individual's feeling good about themselves or self-esteem depends relationship with mother during the early year. If a person has no positive childhood experiences with his or her mother during the early years, a predisposition to depression may develop [5].

\section{The Cognitive-Behavioral View}

\section{Beck's cognitive-behavioral depression theory}

Beck claims suicidal people are suffering from a kind of helpless thought. Depressive thought about all negative feelings, according to Beck. We prefer to personally take things and assume that there is no hope for the future. Beck suggests that this inferior mentality leads the depressed to more negative life experiences. The depressive, in effect, exhibits more nuanced inferiority [7]. They expect too much from themselves and they believe that there is nothing he or she can do about it and very hopeless about the future. They learned to be helpless all through life. They believed that no matter how much they tried to run away from the problems but it were still going to be present [8]. Substantial research shows that depression tends to occur in families and researchers are working with twins and finding that both twins are likely to develop bipolar depression if they have at least one [9]. 


\section{Psychological Risk Factors}

Psychological beliefs emphasized that late-life depression is the same as earlier life depression. Late-life depression is strongly associated with neuroticism and is associated with genetic functions and indicates that neuroticism can be characterized as a psychological threat to depression and a genetic risk [10]. Rumination style is associated with depression, and one's lifespan distress is thought through passively. Individuals with ruminative depression are more likely to benefit from social assistance programme.

\section{Treatments related to evidence based}

Substantial body of evidence supported the practice of many types of therapy related to cognitive behavioral treatments and Psychotherapy for depressed older adults. Randomized clinical trials have shown similar results for selective serotonin reuptake inhibitors with large effect sizes [11]. Both therapies have a feature of cognitive behavior that addresses the problem of behavioral activity with a strong focus on positive performance. Such approaches are called problem-solving counseling, psychodynamic therapy, and behavioral therapy for life analysis, cognitive behavioral therapy. These behavioral therapies were very successful in coping with the anxiety of older adults. Use Life summary therapy for an older adult differs from the practices of memories and is an empirically performed formal therapy. Treatments that add empirically cognitive psychological approaches to address practical issues with a focus on accessing social and community resources may be more powerful than either approach alone. Interview of cognitive behavioral therapy based on care [12]. To the degree that negative impact mitigation can be seen during this cycle by distancing oneself from depressive kind of feelings and the negative feedback. Multi-component therapy helps reduce the caregiver's depression [13]. However, it is essential with older adults that not to over emphasize the stresses in life rather it is necessary to address the significance of biological risk factors and disengagement in behavioral as contributing to state of depression with the factor of psychosocial context [14]. According to some body of research evidence that effectiveness of aerobic exercise as an alternative or supplementary treatment for depression but preventive measures are needed for those adults at increased risk of depressive disorder. In order to prevent major disorder, the most encouraging preventive treatment may be to treat depressed patients with subsyndromal depressive symptoms.

\section{Discussions and Conclusions}

Empirical literature points out that anxiety are not a common feature of the elderly. Depression in midlife is very common than in later stage in life. While depression in old age is not very serious issue but it can have devastating consequences. Risk and protective factors can be understood from older adults view point on their feelings developmental stress. Biological threats are becoming more evident in human old life or in certain events of life, while psychosocial vulnerability is declining and psychosocial resilience is increasing. More research is needed as to identify the major reasons for the onset of major depressive disorders among elderly. The age-old depression etiological picture is very complex. Comorbidity, which results from social and biological causes, is particularly common in elderly life depression. There is consensus and discussion on risk factors of age-old life depression, including stressful events of life such as physical illness and deprivation. To analyze the associations between these risk factors, work already been in progress [1]. As risk factors knowledge accumulates, it becomes possible to design preventive strategies. Emerging work promotes the use of somatic and emotional behavioral interventions to reduce depression in the elderly, including cognitive behavioral and psychosocial treatments for older adults [1].

\section{References}

1. Fiske A, Wetherill JL, Gatz M (2009) Depression in older adults. ARCP 5: 363-389.

2. Elkin I (1994) The NIMH treatment of depression collaborative research program: Where we began and where we are. American Psychological Association.

3. Egede LE, Zheng D, Simpson K (2002) Comorbid depression is associated with increased health care use and expenditures in individuals with diabetes. Diabetes Care 25: $464-470$.

4. Bhui K, Stansfeld S, Hull S, Priebe S, Mole F, et al. (2003) Ethnic variations in pathways to and use of specialist mental health services in the UK. Br J Psychiatry 182: 105-116.

5. Blom MB, Spinhoven $P$, Hoffman $T$, Jonker K, Hoencamp E, et al. (2007) Severity and duration of depression, not personality factors, predict short term outcome in the treatment of major depression. J Affect Disord 104: 119-126.

6. Chew-Graham CA, Hogg T (2002) Patients with chronic physical illness and co-existing and co-existing psychological morbidity: GPs' views on their role in detection and management. Primary Care Psychiatry 8: 35-39.

7. Jane Cahill, Michael Barkham, Gillian Hardy, Anne Rees, David A Shapiro, et al. (2003) Outcomes of patients completing and not completing cognitive therapy for depression. Br J Clin Psychol 42: 133-143.

8. Caroline Burgess, Victoria Cornelius, Sharon Love, Jill Graham, Michael Richards, et al. (2005) Depression and anxiety in women with early breast cancer: Five year observational cohort study. BMJ 330: 702-705.

9. Jayati Das Munshi, Rob Stewart, Khalida Ismail, Paul E Bebbington, Rachel Jenkins, et al. (2007) Diabetes, common mental disorders, and disability: Findings from the UK National Psychiatric Morbidity Survey. Psychosom Med 69: 543-550.

10. Filippo Bogetto, Silvio Bellino, Raffaele Bonatto Revello, Luca Patria (2002) Discontinuation syndrome in dysthymic 
patients treated with selective serotonin reuptake inhibitors: A clinical investigation. CNS Drugs 16: 273-283.

11. Ciechanowski PS, Katon WJ, Russo JE (2000) Depression and diabetes: Impact of depressive symptoms on adherence, function and costs. Arch Intern Med 160: 3278-3285.

12. Paul McCrone, Martin Knapp, Judith Proudfoot, Clash Ryden, Kate Cavanagh, et al. (2004) Cost-effectiveness of computerised cognitive behavioural therapy for anxiety and depression in primary care: Randomised controlled trial. $\mathrm{Br}$ J Psychiatry 185: 55-62.

13. Andrews G, Anderson TM, Slade T, Sunderland M (2008) Classification of anxiety and depressive disorders: Problems and solutions. Depress Anxiety 25: 274-281.

14. Prasher VP (1999) Presentation and management of depression in people with learning disability. APT 5: 447-454. 